\title{
Design and Analysis of a Low Noise Amplifier with matching Networks
}

\author{
B.Sekharbabu ${ }^{1}$, G.Narendra ${ }^{2}$ \\ ${ }^{1}$ Assistant professor, \\ ${ }^{2}$ Assistant Professor, \\ ${ }^{1}$ ECE Department, Vardhaman College of Engineering, Hyderabad, India \\ ${ }^{2}$ ECE Department, Vignan's Institute of Management and Technology for Women, Hyderabad, India
}

\begin{abstract}
An electronic equipment will increase the ability of each the signal and also the noise gift at its input. However there square measure disadvantages with the increasing noise power throughout amplification. Therefore, there was a desire for various such signals ought to be amplified maintaining noise power constant. Hence, LNAs square measure designed to attenuate further noise. Low-noise electronic equipment (LNA) is electronic electronic equipment that amplifies an awfully low-power signal while not considerably degrading its $S N R$ value. Noise will be reduced by considering electrical resistance matching, selecting the electronic equipment technology (such as low-noise components), and choosing low-noise biasing conditions. LNAs square measure found at the receiving finish of nearly each communications system like radio communication systems, medical instruments. A typical LNA could provide an influence gain 20 $d B$ whereas decreasing the signal/noise ratio by but an element of 2 (a three decibel noise figure (NF)) its performance is measured in an exceedingly variety of figures of advantage. We'll be planning noise electronic equipment that operates at $0.9 \mathrm{GHz}$ and expected to own a $G_{\max }$ of $12.69 \mathrm{~dB}$ and smith chart is employed to form matched circuits to attain the expected gain. Here we have a tendency to attempt to accomplish electrical resistance matching victimization Smith tool that could be an elementary aid in electrical resistance matching network style and additionally is a typical for graphical presentation of electrical resistance, reactance, stability circles, gain circles, noise circles with the assistance of laptop -aided ANSYS HFSS tool.
\end{abstract}

Keywords: ANSYS HFSS, IMPEDANCE MATCHING, NOISE FIGURE, RETURN LOSS, SMITH TOOL

\section{Introduction}

Antennas are one of the common sources of weak signals. ${ }^{[1]}$ An outdoor antenna is often connected to its receiver by a transmission line called a "feed line". Any losses in the feed line adversely affect the received signal-to-noise ratio: a feed line loss of $3 \mathrm{~dB}$ degrades the signal-to-noise ratio (SNR) by $3 \mathrm{~dB}$. An example is a feed line made from 10 feet $(3.0 \mathrm{~m})$ of RG-174 coaxial cable and uses a global positioning system (GPS) receiver. The loss in that feed line is $3.2 \mathrm{~dB}$ at 1 gigahertz $(\mathrm{GHz})$; approx. $5 \mathrm{~dB}$ at the GPS frequency $(1.57542 \mathrm{GHz})$. This feed line loss can be avoided by placing an LNA at the antenna, which supplies enough gain to offset the feed line loss. A good LNA has a low NF (e.g. $1 \mathrm{~dB}$ ), enough gain to boost the signal (e.g. 10 $\mathrm{dB}$ ) and a large enough inter-modulation and compression point (IP3 and P1dB) to do the work required of it. Further criteria are the LNA's operating bandwidth, gain flatness, stability, input, and output voltage standing wave ratio (VSWR).For low noise, a high amplification is required for the amplifier in the first stage. Therefore, junction field-effect transistors (JFETs) and high electron mobility transistors (HEMTs) are often used. They are driven in a high-current regime, which is not energy-efficient, but it reduces the relative amount of shot noise. It also requires input and output matching circuits for narrow-band circuits to enhance the gain (see Gainbandwidth product).

\section{Noise reduction}

An LNA is a key component at the front-end of a radio receiver circuit to help reduce unwanted noise in particular. Friis' formulas for noise models the noise in a multi-stage signal collection circuit. In most receivers, the overall noise figure (NF) is dominated by the first few stages of the receiver's 'front-end'.

By using an LNA close to the signal source, the effect of noise from subsequent stages of the receive chain in the circuit is reduced by the signal gain created by the LNA, while the noise created by the LNA itself is injected directly into the received signal. The LNA boosts the desired signals' power while adding as little noise and distortion as possible. The work done by the LNA enables optimum retrieval of the desired signal in the later stages of the system. 


\section{A. LNA design:}

\section{Designing Procedure}

Low noise amplifiers are the building blocks of communication systems and instruments. The four important parameters in LNA design are: gain, noise figure, non-linearity and impedance matching. The steps required in designing an LNA are:

\section{1) Gain device}

Amplifiers need a device to provide gain. In the 1940s, that device was a vacuum tube, but now it is usually a transistor or an integrated circuit. The transistor may be one of many varieties of bipolar transistors or fieldeffect transistors. Other gain devices, such as tunnel diodes, may be used.

Broadly speaking, there are two categories of transistor models used in the design of low-noise amplifiers:

- Small-signal models use quasi-linear models to model noise.

- Large-signal models that consider non-linear mixing.

\section{2) Circuit topology}

Circuit topology covers issues such as gain and input impedance.

Gain is often a compromise. On one hand, having lots of gain is good because it takes weak signals above the noise floor. On the other hand, lots of gain means higher level signals and more problems with non-linear mixing. The circuit topology also affects input and output impedance. In general, the source impedance is matched to the input impedance because that will maximize the power transfer from the source to the device. If the source impedance is low, then a common base or common drain circuit topology may be appropriate. For medium source impedance, then a common or common source topology may be used. With a high source resistance, then a common collector or common drain topology may be appropriate. An input impedance match may not produce the lowest noise figure. There is another notion of a noise impedance match. Another design issue is the noise introduced by biasing networks.

\section{LNA With Different Combinations}

The signals delivered by the antenna in modern communication systems can be in the range of very low power in this case there is a need of increasing the power level. This intention makes the use of Low noise power amplifier which can amplify the signal and as well as reduce the noise there are several mechanisms for designing of low noise amplifier but in this paper LNA is designed using a transistor with the model number $\left(\mathrm{ne} 68133, \mathrm{~V}_{\mathrm{c}}=2.5 \mathrm{v}, \mathrm{I}_{\mathrm{c}}=3 \mathrm{~mA}\right)$.

Here the simulations are carried out for three cases

1. LNA without degenerative inductor and matched network

2. LNA with degenerative inductor and without matched network

3. LNA with degenerative inductor and matched network

\section{LNA without degenerative inductor and matched network}

In the first case low noise amplifier is designed without connecting a degenerative inductor to the emitter terminal of the transistor and matching networks at input and load side the equivalent model for this case is given in figure 1 .

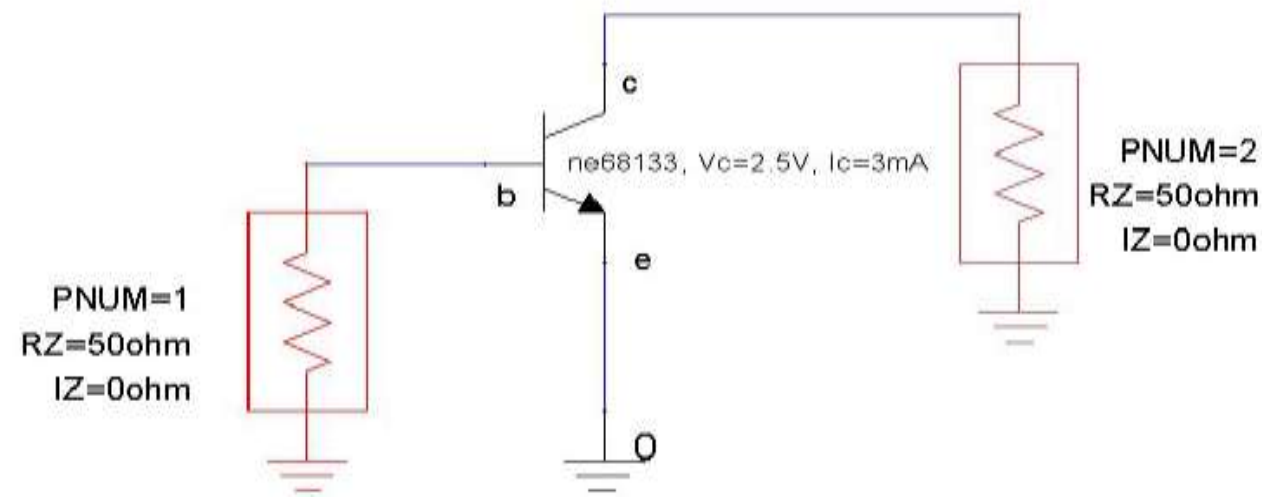

Fig 1 .low noise amplifier without degenerative inductor and matched networks 


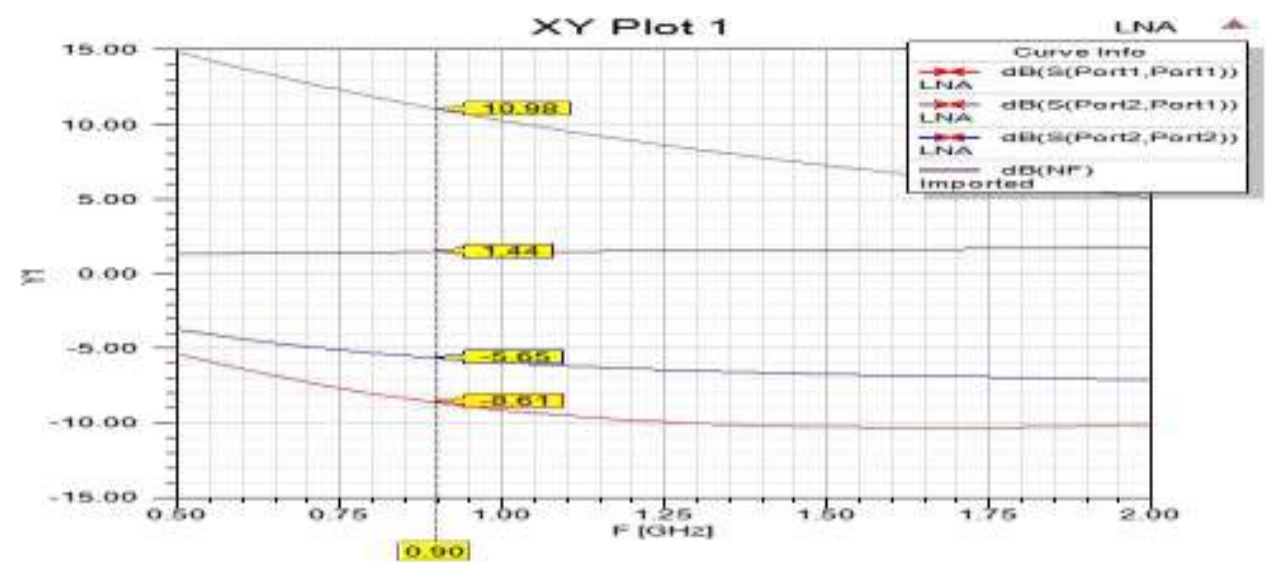

Fig 2 .Return Loss, Insertion Loss, and Noise Figure for LNA under case: 1

Because of improper impedance matching at input side and load side the return loss values at port 1 and port are very high and because of absence of degenerative inductor the transistor lost its stability. In this case at port 1 the return loss value is around $-8 \mathrm{~dB}$ and at port 2 the return loss value is around $-5 \mathrm{~dB}$. So there is a requirement of connection of inductor in between emitter terminal of transistor and ground, this idea leads to second case.

\section{LNA with degenerative inductor and without matched network}

In the second case a degenerative inductor with a value of $1.4 \mathrm{nH}$ is connected in between emitter and ground. The degenerative inductor will stabilize the transistor and also avoids reflections at input and output ports. The equivalent model is shown in figure 2.

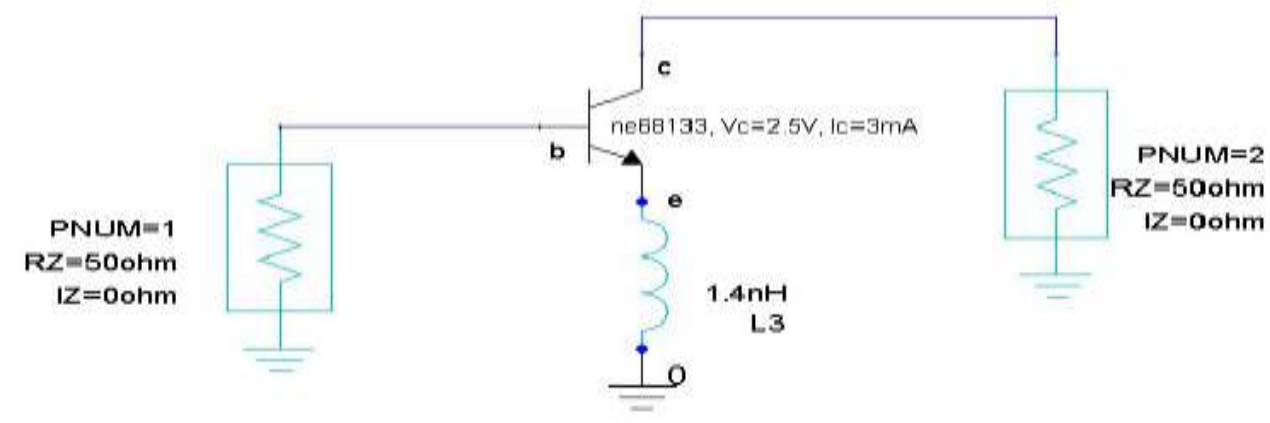

Fig 3 .Low noise amplifier with degenerative inductor and without matched networks

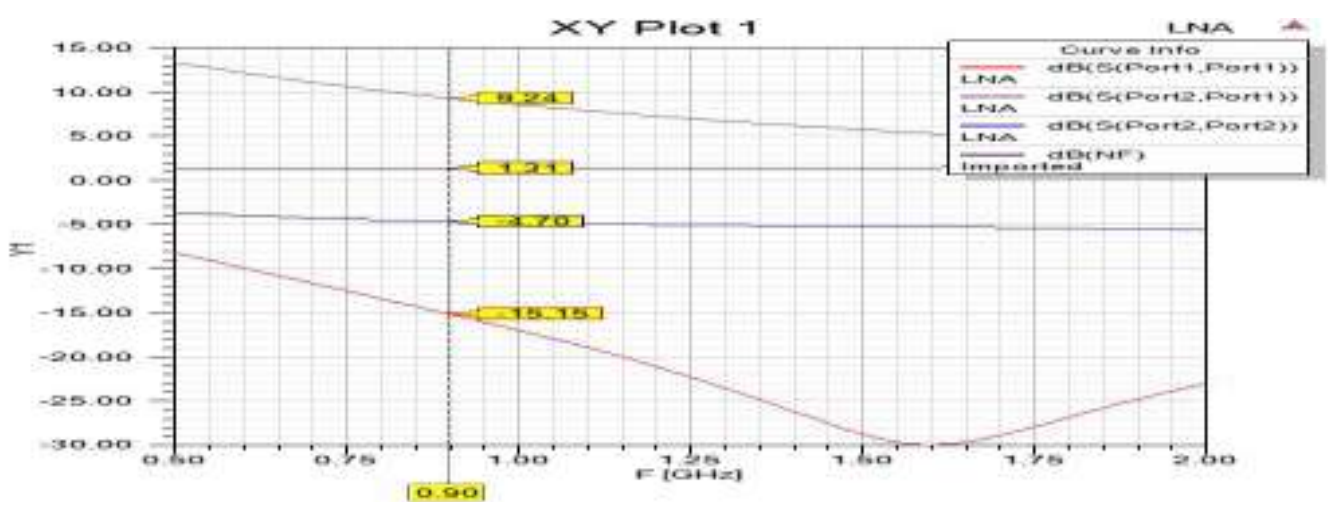

Fig 4 .Return Loss, Insertion Loss, and Noise Figure for LNA under case: 2

In this case at port 1 the return loss value is around $-15 \mathrm{~dB}$ and at port 2 the return loss value is around $-5 \mathrm{~dB}$. So there is a requirement of connection of inductor in between emitter terminal of transistor and ground and also matching networks at input and output ports this idea leads to third case. 


\section{LNA with degenerative inductor and without matched network}

In the third case a degenerative inductor with a value of $1.4 \mathrm{nH}$ is connected in between emitter and ground and two matched networks are connected at source and load side. The degenerative inductor and matching networks will stabilize the transistor and also avoids reflections at input and output ports. In the matching network the values of lumped elements are calculated by using smith tool after so many iteration the matched networks are finalized with a combination of capacitor and inductor. The equivalent models for matching networks are shown in figure $5 \&$ figure 6.

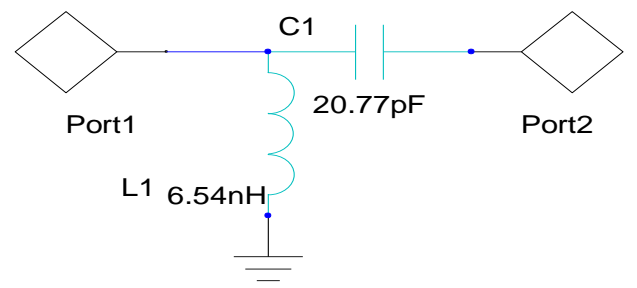

Fig 5.input side matching network

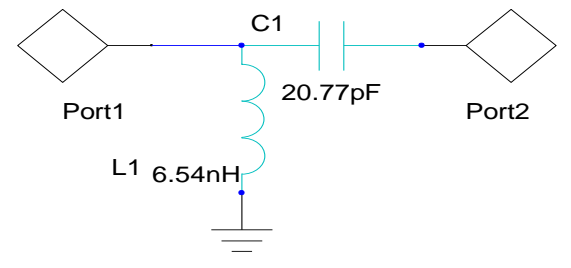

Fig 6.output side matching network

In the input matching network an inductor with a value of $6.54 \mathrm{nH}$ is connected in series with a capacitor of having capacitance of $20.77 \mathrm{pF}$ to match the source. In the output matching network an inductor with a value of $13.31 \mathrm{nH}$ is connected in series with a capacitor of having capacitance of $1.37 \mathrm{pF}$ to match the load. These two matching networks are added to the second case and the equivalent model is shown in figure 7.

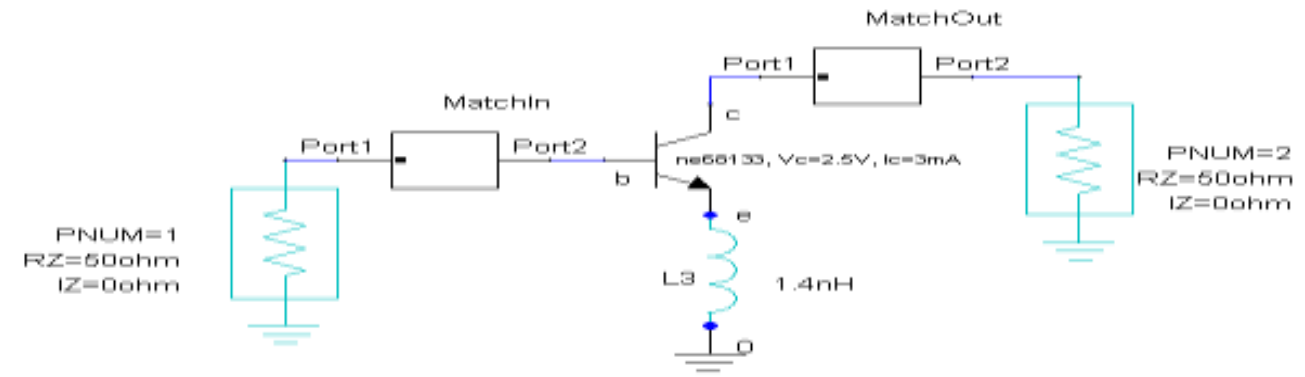

Fig 7 .Low noise amplifier with degenerative inductor and matched networks

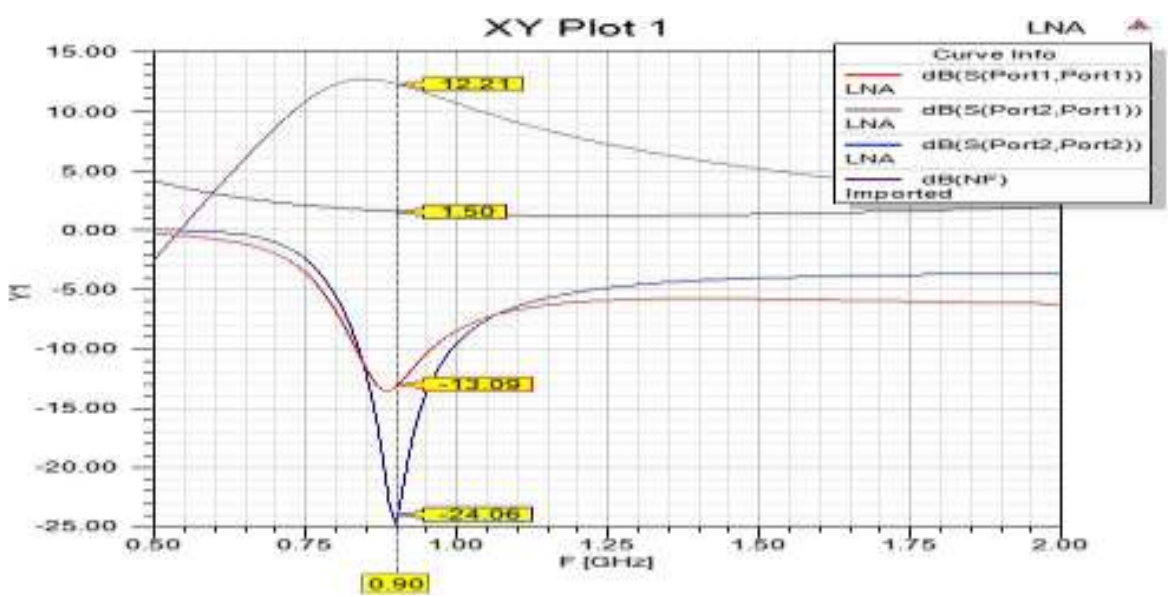

Fig 4 .Return Loss, Insertion Loss, and Noise Figure for LNA under case: 2

In this case the return loss values at both the ports are very less at port 1 the return loss value is around $-13 \mathrm{~dB}$ and at port 2 the return loss value is around $-24 \mathrm{~dB}$. The insertion value between port 1 and port is $12.21 \mathrm{~dB}$ and the noise figure value is $1.5 \mathrm{~dB}$. These are the effective parameters for the low noise amplifier which is operating at $0.9 \mathrm{GHz}$. The smith chart which will give the value of reflection co-efficient is shown in figure. Here the reflection co-efficient for all three cases is calculated and compared with other model. The reflection coefficient for first case is around 0.3 , second case is 0.21 and for third case is 0.17 


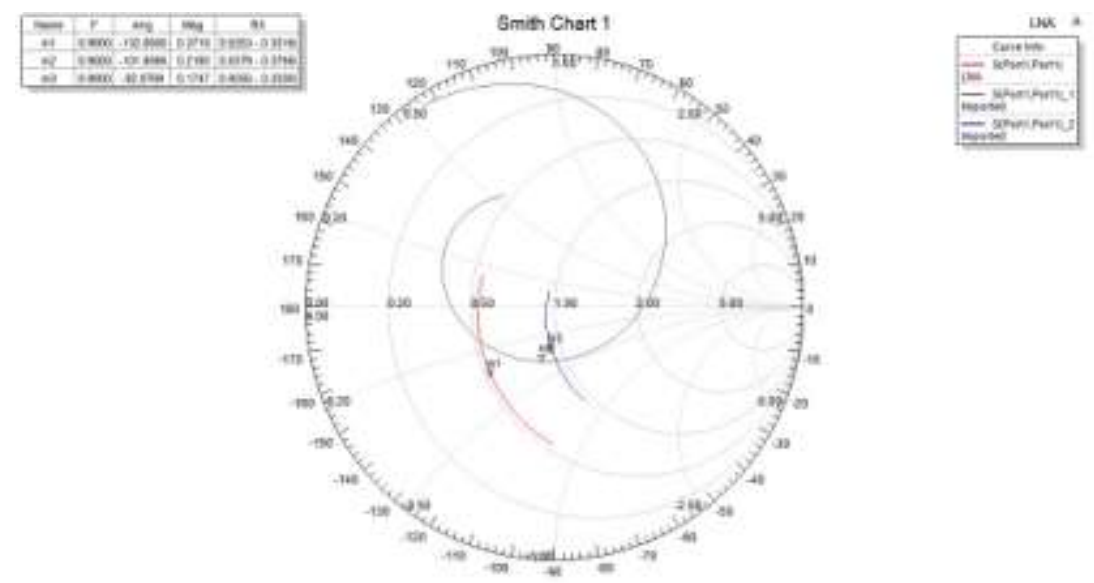

Fig 9.Comparison of reflection coefficients for all three cases using smith chart

Table 1.comparison of LNA simulation results for all three cases

\begin{tabular}{|l|l|l|l|l|l|}
\hline \multirow{2}{*}{ Low noise amplifier } & \multicolumn{2}{|l|}{ Return Loss(dB) } & Insertion loss(dB) & $\begin{array}{l}\text { Noise } \\
\text { figure }(\mathrm{dB})\end{array}$ & Reflection co-efficient \\
\cline { 2 - 5 } & at Port 1 & at Port 2 & & 1.44 & 0.37 \\
\hline With Out L3\& MT & -8.61 & -5.65 & 10.98 & 1.31 & 0.21 \\
\hline With L3 \& Without MT & -15.15 & -4.70 & 9.24 & 1.5 & 0.17 \\
\hline With L3 \& MT & -13.09 & -24.06 & 12.21 & \\
\hline
\end{tabular}

L3.....De-generative inductor

MT.........Matching network

\section{Conclusions}

In this paper a low noise amplifier is designed and the simulation results obtained during this investigation demonstrate a promising new approach to provide impedance matching and amplification. The Designed LNA provides Very Less Return Loss at Port 1 and Port2 and Also it provide a noise figure of $1.5 \mathrm{~dB}$. Thus, the proposed LNA is tiny solution for applications such as industrial, scientific and medical band (ISM) radios, cellular telephones, GPS receivers, cordless phones, wireless LANs (Wi-Fi), automotive remote, and satellite communications

\section{Acknowledgements}

The authors would like to acknowledge the support extended by the following as the proposed research work has been carried out in RF-HFSS Laboratory, Vardhaman College of Engineering funded by Department of Science \& Technology, Govt. of India.

\section{Journal Papers:}

\section{References}

[1] Molavi, R.; Mirabbasi, S.; Hashemi, M., "A wideband CMOS LNA design approach, "Circuits and Systems, 2005. IEEE International Symposium on, vol., no., pp.5107,5110 Vol. 5, 23-26May 2005

[2] Qiang Li; Yue Ping Zhang.,"A 1.5-V 2-9.6-GHz Inductor less Low- Noise amplifier in 0.13 4 m CMOS",IEEE Transactions On Microwave Theory and Techniques, Vol. 55, No. 10, pp 2015-2023, October 2007.

[3] Yu-Tsung Lo; Jean-Fu Kiang., "Design of Wideband LNAs Using Parallel to Series Resonant Matching Network between Common- Gate and Common-Source Stages",IEEE Transactions On Microwave Theory And Techniques, Vol. 59, No. 9, pp 22852294, September 2011.

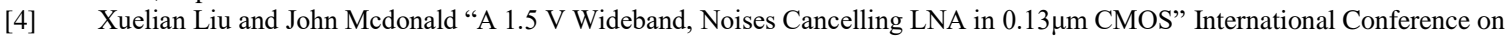
Solid-State and Integrated Circuit (ICSIC 2012) IPCSIT vol 32. Page 45-50.

[5] Nam Hwi Jeong, Choon Sik Cho and Seungwook Min "Highly Linear wideband LNA Design Using Inductive Shunt Feedback"

\section{Books:} Journal of Semiconductor Technology and Science, Vol. 14, No.1, February 2014

[1]. "Micro wave and RF engineering", volume 1. An electronic design automation approach by Ali bhegai, Stephen turner.

[2]. "Micro Wave Transistor Amplifiers Analysis and design" second edition by Guillerno Gonzalez.

[3]. . . .RF Circuit design" by Chris bowick, Jhon blyer, Cheryl ajluni. 